\title{
Multifractal analysis of high resolution solar wind proton density measurements
}

\author{
Luca Sorriso-Valvo ${ }^{\mathrm{a}, *}$, Francesco Carbone ${ }^{\mathrm{b}}$, Ersilia Leonardis ${ }^{\mathrm{c}}$, \\ Christopher H. K. Chen ${ }^{d}$, Jana Šafránkováe ${ }^{\mathrm{e}}$ Zdenek Němeček ${ }^{\mathrm{e}}$ \\ ${ }^{a}$ CNR-Nanotec - Institute of Nanotechnologies, U.O.S. di Cosenza, Ponte P. Bucci, cubo \\ 31c, 87036 Rende, Italy \\ ${ }^{b}$ CNR-IIA - Institute of Atmospheric Pollution Research, U.O.S. di Rende, c/o: \\ UNICAL-Polifunzionale, 87036 Rende, Italy \\ ${ }^{c}$ Dipartimento di Fisica, Università della Calabria, Ponte P. Bucci, cubo 31C, 87036 \\ Rende, Italy \\ ${ }^{d}$ Department of Physics, Imperial College London, London SW7 2AZ, UK \\ ${ }^{e}$ Faculty of Mathematics and Physics, Charles University, Prague 18000, Czech Republic
}

\begin{abstract}
The solar wind is a highly turbulent medium, with a high level of field fluctuations throughout a broad range of scales. These include an inertial range where a turbulent cascade is assumed to be active. The solar wind cascade shows intermittency, which however may depend on the wind conditions. Recent observations have shown that ion-scale magnetic turbulence is almost self-similar, rather than intermittent. A similar result was observed for the high resolution measurements of proton density provided by the spacecraft Spektr-R. Intermittency may be interpreted as the result of the multifractal properties of the turbulent cascade. In this perspective, this paper is devoted to the description of the multifractal properties of the high resolution density measurements. In particular, we have used the standard coarse-graining technique to evaluate the generalized dimensions $D_{q}$, and from these the multifractal spectrum $f(\alpha)$, in two ranges of scale. A fit with the p-model for intermittency provided a quantitative measure of multifractality. Such indicator was then compared with alternative measures: the width of the multifractal spectrum, the peak of the kurtosis, and its scaling exponent. The results indicate that the small-scale fluctuations are multifractal, and
\end{abstract}

\footnotetext{
*Corresponding author

Email address: sorriso@fis.unical.it (Luca Sorriso-Valvo)
} 
suggest that different measures of intermittency are required to fully understand the small scale cascade.

Keywords: Solar Wind, Turbulence, Multifractal.

\section{Introduction}

The solar wind is a rarefied, supersonic and super-Alfvénic flow of particles travelling away from the Sun, and permeating the whole heliosphere. Since the advent of the space era, several space missions have been devoted to the measurement of the solar wind parameters and fields, which represent a unique opportunity for the in-situ experimental study of astrophysical plasmas (Bruno \& Carbone, 2013). One of the main characteristics of solar wind is turbulence (Frisch, 1995). Ubiquitously observed in neutral fluid and plasma flows, turbulence is the way that allows the transfer of energy from large scales, where it is normally provided, down to microscopic scales, where dissipative and dispersive processes can efficiently convert it, for example, into heat or accelerated particles. The turbulent transfer of energy through scales is described by the nonlinear terms of the dynamic equations (such as Navier-Stokes for neutral flows or magnetohydrodynamics for collisional plasmas). This term is responsible for the transfer of energy across different wave vectors, resulting in an energy cascade from large-scale to small-scale fluctuations. Such transfer is easily observed through the spectral properties of the field fluctuations, which display a power-law scaling in the so-called inertial range, where the nonlinear interactions dominate over the dissipative processes. In the Kolmogorov phenomenology of turbulence (Kolmogorov, 1941), a spectral index can be predicted which may depend on the specific physical processes of each different system. The power-law behavior is the result of scale invariance and self-similarity of the system. A power-law spectrum was observed in the solar wind magnetic fluctuations since the early space observations (Coleman, 1968), and is nowadays a well established feature (see e.g. Bruno \& Carbone (2013) and references therein). Alongside the power-law spectrum, another important characteristic of turbulence is intermittency (Frisch, 1995). This term was introduced to account for the inhomogeneity of the energy transfer process, which leads in turn to inhomogeneity of the energy dissipation. Intermittency can be quantitatively measured through the scale-dependence of the statistical properties of the field fluctuations. Indeed, the inhomogeneity of the energy transfer results in 
the concentration of energy in small regions of space, which produces highly energetic fluctuations. Correspondingly, the tails of the probability distribution functions (PDFs) rise as the scale decreases, being populated more and more by such energetic, intermittent structures. This breaks the selfsimilarity and produces the anomalous scaling of the statistical properties of the field fluctuations (Anselmet et al., 1984; Frisch, 1995). Self-similar processes, characterized by the singularity of a suitably defined probability measure, are often described in terms of the fractal properties of the system (Mandelbrot, 1982). Similarly, the anomalous scaling of an intermittent turbulent field is associated to the multifractal nature of the cascade (Frisch \& Parisi, 1983; Benzi et al., 1984; Frisch, 1995). Multifractality (and thus intermittency) of a system can be quantitatively described, for example, by the whole set of their generalized dimensions, or equivalently by their singularity spectrum (see e.g. Halsey et al. (1986); Ott (1993)).

Intermittency has been deeply studied in the solar wind inertial range ( $\mathrm{Tu}$ \& Marsch, 1995; Marsch \& Tu, 1997; Sorriso-Valvo et al., 1999, 2001; Bruno \& Carbone, 2013), and more recently in the ion range of scales (Alexandrova et al., 2008; Kiyani et al., 2009). Experimental evidence shows that both velocity and magnetic field are strongly intermittent, with formation of small scale vortexes, current sheets, and other type of structures (Bruno \& Carbone, 2013). Although the solar wind plasma is often considered as nearly incompressible, data analysis has shown that the proton density also has Kolmogorov-like power spectra (Montgomery et al., 1987; Marsch \& Tu, 1990; Riazantseva \& Zastenker, 2008; Shaikh \& Zank, 2010; Chen et al., 2011). Moreover, the study of higher order statistics indicates that density is highly intermittent, with scale-dependent statistical properties and formation of small scale structures (Hnat et al., 2003, 2005). Recent results have shown that inertial range density intermittency decreases when the wind travels from approximately Mercury's to the Earth's orbit (Bruno et al., 2014). The reason for this radial evolution is unclear, but is consistent with the parametric decay of Alfvénic fluctuations originating in the solar corona (Malara et al., 2001; Primavera et al., 2003; Bruno et al., 2014). Density fluctuations have also been studied in the sub-proton gyro-radius range of scales, where a steepening of the spectrum was observed (Chen et al., 2012; Šafránková et al., 2013a). Furthermore, in the small-scale range the statistical properties of the density fluctuations do not vary significantly with the scale (Chen et al., 2014). Similar features were previously observed in small scale magnetic field fluctuations (Kiyani et al., 2009, 2013; Wu et al., 2013), although diffrent 
solar wind samples may instead show enhanced intermittency (this is qualitatively shown in Alexandrova et al. (2008) where, however, quantitative results could be affected by data calibration errors).

The multifractal properties of the heliospheric magnetic field (Burlaga, 1991, 1992; Macek, 2006, 2007) and velocity (Marsch et al., 1996; Macek \& Szczepaniak, 2008) have been deeply studied, showing the multifractal nature of solar wind turbulence and its connection with intermittency. The multifractal approach has been used, for example, to characterize the evolution of heliospheric turbulence in its radial expansion (Burlaga, 2004, 2006; Burlaga \& Ness, 2010; Macek \& Wawrzaszek, 2009; Macek et al., 2011, 2012), or to describe intermittency in the magnetospheric cusp (Yordanova et al., 2004), near the heliopause (Macek et al., 2014) and beyond the ecliptic at high latitudes (Wawrzaszek et al., 2015). An explicit link between solar wind intermittency and the multifractal cascade (Frisch \& Parisi, 1983; Benzi et al., 1984; Castaing et al., 1990) has been recently pointed out (Sorriso-Valvo et al., 2015).

In this paper we investigate the small scale properties of turbulence through the multifractal analysis of solar wind proton density time series recorded by the spacecraft Spektr-R. In Section 2 we describe the solar wind dataset used for this work; Section 3 is devoted to the description of the analysis technique and of the results; final considerations are collected in Section 4.

\section{Solar wind data: the Spektr-R proton density}

The data used for this work are the high time resolution solar wind proton density $n_{p}$ measured by the BMSW instrument (Šafránková et al., 2013b) on the Spektr-R spacecraft. The six Faraday cups of BMSW can sample the solar wind ion distribution at $f_{0}=32$ samples/s (i.e. at $0.031 \mathrm{~s}$ resolution), along with the proton velocity $v_{p}$ and temperature $T_{p}$. Since Spektr-R does not provide magnetic field measurements, the kinetic scales and proton plasma $\beta_{p}$ were estimated using the magnetic field $B$ measured by MFI on Wind in the corresponding time interval (Chen et al., 2014). The data under study consist of eight samples, each spanning 1 to 4 hours, extracted from four larger samples already studied in previous works (Šafránková et al., 2013a,b; Chen et al., 2014; Šafránková et al., 2015), and all measured between November 2011 and August 2012. The subsamples were chosen in order to completely avoid large data gaps ( $>0.8$ seconds) and to ensure a better statistical stationarity. Short data gaps (totalling less than $1 \%$ of each 
Table 1: List of the eight intervals parameters. All data are from Spektr-R, except $B$, which is from the upstream Wind spacecraft. For each sample, the Date (dd/mm/yyyy) refers to the initial time of the measurement. Time is in UT, magnetic field $B$ is in nT, the proton density $n_{p}$ is in $\mathrm{cm}^{-3}$, and the proton speed $v_{p}$ in $\mathrm{km} \mathrm{s}^{-1}$.

\begin{tabular}{ccccccc}
\hline \hline Interval & Date & Time & $B$ & $n_{p}$ & $v_{p}$ & $\beta_{p}$ \\
\hline$A$ & $10 / 11 / 2011$ & $15: 55: 40-18: 46: 55$ & 4.7 & 4.6 & 370 & 0.78 \\
$B$ & $01 / 06 / 2012$ & $21: 05: 44-01: 09: 06$ & 8.3 & 6.6 & 370 & 0.12 \\
$C$ & $02 / 06 / 2012$ & $02: 34: 52-03: 26: 43$ & 9.1 & 7.9 & 360 & 0.17 \\
$D$ & $02 / 06 / 2012$ & $06: 02: 22-08: 07: 15$ & 8.8 & 8.2 & 330 & 0.18 \\
$E$ & $09 / 07 / 2012$ & $08: 25: 56-11: 09: 51$ & 12.0 & 6.0 & 400 & 0.06 \\
$F$ & $09 / 07 / 2012$ & $13: 22: 18-16: 55: 40$ & 11.0 & 6.7 & 390 & 0.14 \\
$G$ & $09 / 08 / 2012$ & $10: 48: 52-15: 59: 13$ & 4.7 & 4.0 & 320 & 0.74 \\
$H$ & $09 / 08 / 2012$ & $17: 40: 39-22: 31: 50$ & 4.5 & 6.3 & 330 & 0.41 \\
\hline
\end{tabular}

sample) were linearly interpolated. The main parameters of the samples, i.e. the mean magnetic field $B$, proton velocity $v_{p}$ and density $n_{p}$, and the proton plasma $\beta_{p}$, are collected in Table 1. Figures from 1 to 4 show the four intervals and the eight selected subsets (top panels), highlighted as yellow shaded area and labeled with a letter from $A$ to $H$.

The spectral properties of Spektr-R data were deeply studied in previous works (Šafránková et al., 2013a,b; Chen et al., 2014; Šafránková et al., 2015), where two power-law frequency spectra $E(f) \propto f^{-\beta}$ were identified, separated by a break located around the proton gyro-scale. In the eight data samples used for this work, a Kolmogorov-like spectrum is found at large scales, with scaling exponent $\beta \simeq 5 / 3$. At smaller scales, a steeper spectrum is generally observed, with more variable exponents $\beta \simeq 1.76-2.86$, consistent with previous observation obtained using broader samples (Šafránková et al., 2015). The spectral exponents for the two ranges are collected in Table 2 for all samples.

\section{Multifractal analysis of solar wind proton density}

A multifractal is characterized by a variable singularity exponent (Paladin \& Vulpiani, 1987), which describes the degree of regularity at a given position or time, whereas a simple fractal is completely described by one single scaling exponent. The multifractal properties of a signal are thus well described by 

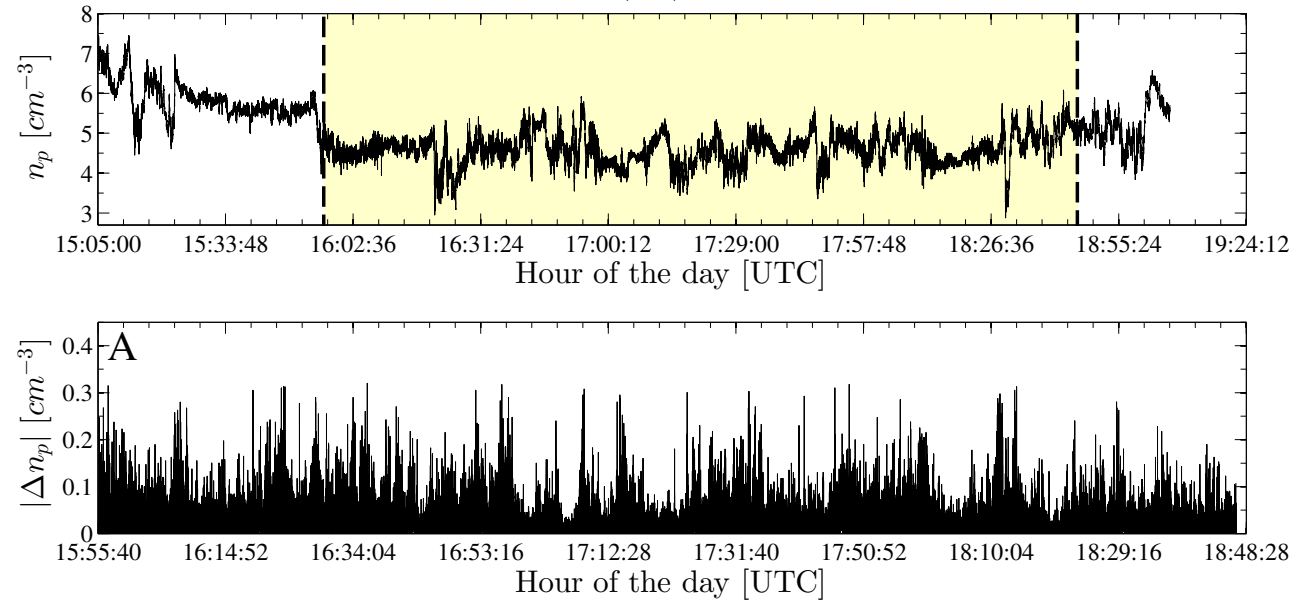

Figure 1: Top panel: the proton density $n_{p}$ as measured by Spektr-R spacecraft on 10/11/2011, from 15:05:00 UT to 19:24:12 UT. Bottom panel: the filtered density gradients $\left|\Delta n_{p}\right|$ in the selected subsample $A$ (highlighted in yellow shade in top panel).
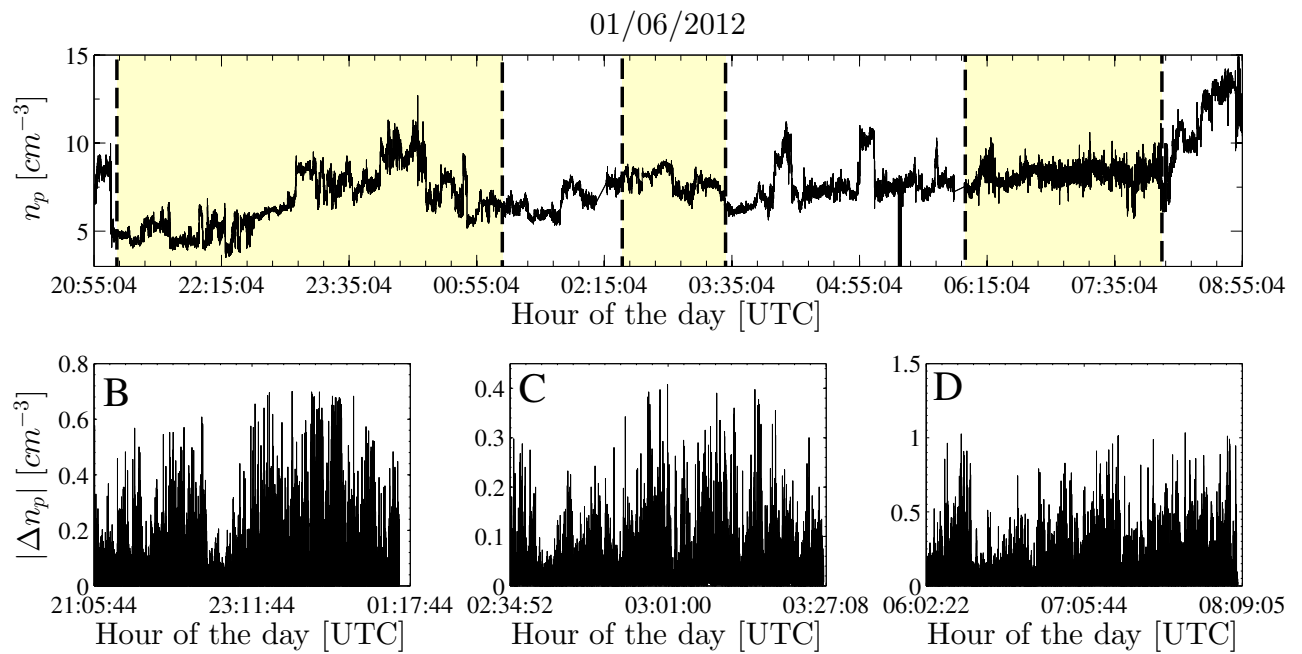

Figure 2: Same as Figure 1 for the day 01/06/2012, from about 20:55:04 UT to 08:55:04 UT (top) and for the subsamples $B, C$ and $D$ (bottom). 
09/07/2012
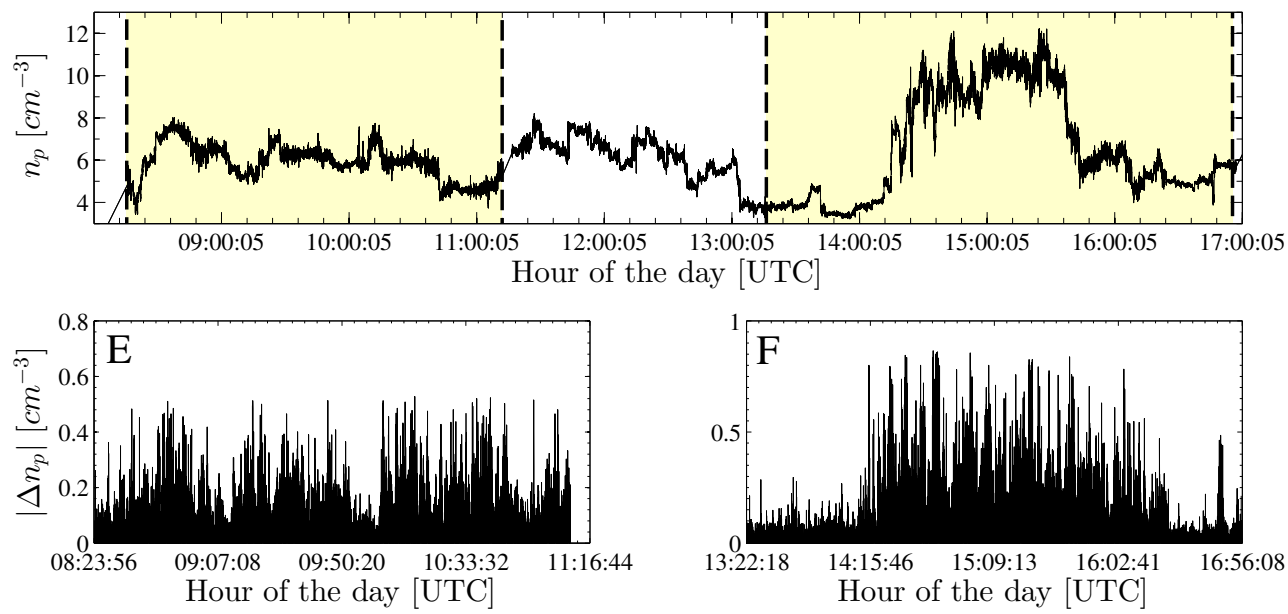

Figure 3: Same as Figure 1 for the day 09/07/2012, from 08:00 UT to 17:00 UT (top) and for the subsamples $E$ and $F$ (bottom).

09/08/2012
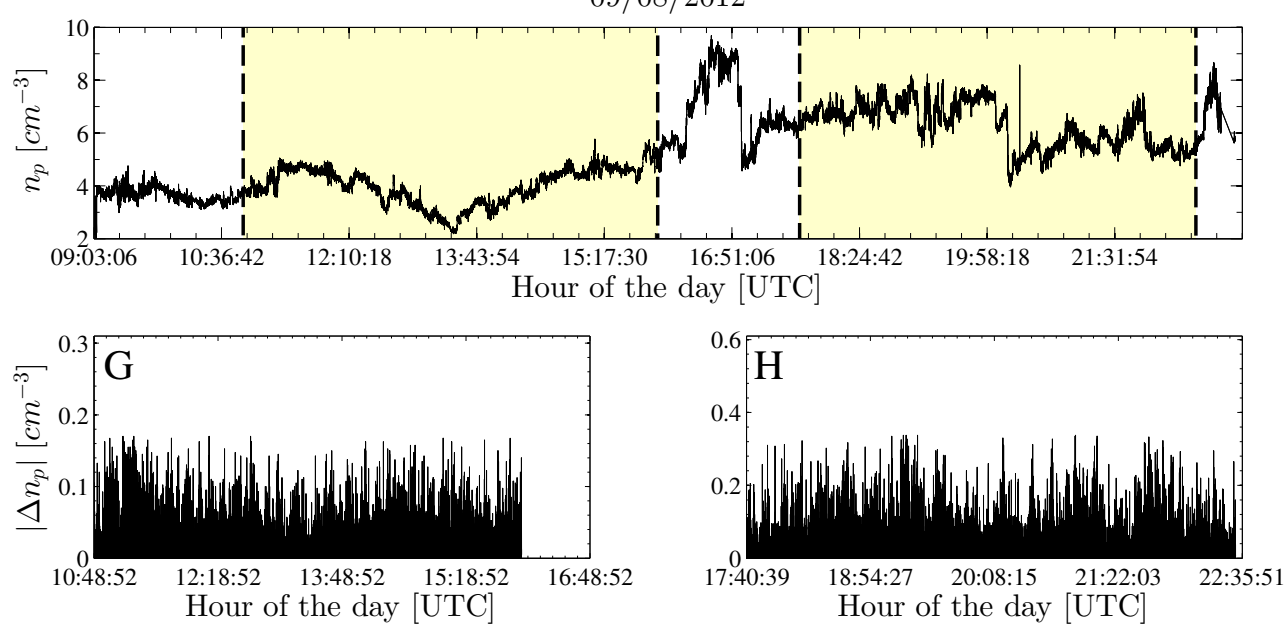

Figure 4: Same as Figure 1 for the day 09/08/2012, from about 09:00 UT to 23:00 UT (top) and for the subsamples $G$ and $H$ (bottom). 
the so-called multifractal spectrum, a quantitative estimate of such variability (Paladin \& Vulpiani, 1987). The broader the multifractal spectrum, the more intense the multifractality (as opposed to "mono"-fractality). For the estimation of the multifractal spectrum of the solar wind proton density, we use the classical approach based on the scaling properties of the associated probability measure (Paladin \& Vulpiani, 1987; Chhabra et al., 1989; Macek et al., 2012; Leonardis et al., 2016).

The first step for the multifractal analysis is the definition of the physical quantity to be studied. In our case, in analogy with previous studies of solar wind magnetic field (Macek et al., 2012), we focus on the proton number density increments $\Delta n_{p}(t)=\left|n_{p}(t+d t)-n_{p}(t)\right|$, where $n_{p}(t)$ is the time series of the proton number density in $\mathrm{cm}^{-3}$ and $d t$ is the data resolution in seconds. As already done for previous analysis of intermittency on similar data (Kiyani et al., 2006; Chen et al., 2014), in order to ensure a better statistical consistency, all the outliers, identified here as the density gradients larger than ten standard deviations, have been discarded. We have checked that similar results hold when using the whole dataset, or when removing outliers with different thresholds. The resulting time series $\Delta n_{p}$ is depicted in the bottom panels in Figures 1-4, where the bursty, intermittent behavior of the field gradient is evident, as expected at those scales (Chen et al., 2014).

Given the time series $\Delta n_{p}(i), i=\{1, \ldots, N\}$ consisting of $N$ data points, the singularity properties can be explored by partitioning the data into scaledependent subsets, and then performing a coarse-graining of a suitably defined probability measure. In order to do so, the time series is partitioned in disjoint subsets of variable time scale $\Delta t$, with $j=\{1, \ldots, T / \Delta t\}, T$ being the sample duration. For each subset, the scale dependent local probability measure can be estimated as:

$$
\mu_{j}(\Delta t)=\frac{\sum_{i=(j-1) \Delta t+1}^{j \Delta t}\left|\Delta n_{p}(i)\right|}{\sum_{i=1, N}\left|\Delta n_{p}(i)\right|} .
$$

Here the sum in numerator includes all the data points within the $j$-th box of size $\Delta t$, and the denominator is a normalization factor computed over the whole dataset. Then, the presence of singularities is revealed by the power-law scaling of the partition functions, defined as:

$$
\chi_{q}(\Delta t)=\sum_{j=1}^{M} \Delta t\left[\mu_{j}(\Delta t)\right]^{q} \propto(\Delta t)^{\tau_{q}},
$$


where $M$ is the scale-dependent number of boxes. Note that the scaling exponents $\tau(q)$ are more rigorously defined as

$$
\tau_{q}=\lim _{\Delta t \rightarrow 0} \frac{-\log \chi_{q}(\Delta t)}{\log \left(\Delta t^{-1}\right)} .
$$

The partition functions carry information on the type of singularities, by distorting and amplifying (via the power $q$ ) the "mass" features of the field and its scaling properties. The set of exponents $\tau_{q}$ describes the singularities, and thus the multifractal properties of the field. In particular, exponents are expected to depend linearly on the order $q$ for mono-fractal objects, where only one singularity exponent is present. On the contrary, deviation from linearity indicates multifractality, i.e. a broader set of singularity exponents.

The set of generalized dimensions $D_{q}$ can then be directly obtained as $D_{q}=\tau_{q} /(q-1)$. The shape and range of variability of $D_{q}$ gives, again, quantitative information on the degree of multifractality of the field. A fractal field should result in a single-valued distribution $D_{q}$, which becomes broader as the degree of multifractality increases. Furthermore, some of the dimensions also have a more specific meaning, for example $D_{0}$ is the Capacity Dimension (also known as "box-counting" dimension), $D_{1}$ is the Information Dimension, and $D_{2}$ the Correlation Dimension (Grassberger \& Procaccia, 1983). An alternative description is defined in terms of the local singularity strength $\alpha$ and the corresponding singularity spectrum $f(\alpha)$, which basically gives the fractal dimensions of the subsets where the field has singularity strength $\alpha$ (Chhabra et al., 1989; Paladin \& Vulpiani, 1987). As for the set of generalized dimensions, the shape of $f(\alpha)$ can be used to quantitatively estimate the degree of multifractality of a field, for example by measuring its width (Macek et al., 2012). The singularity spectrum is related to the scaling exponents $\tau_{q}$ of the partition function, so that it can be easily calculated using the two relations $\alpha_{q}=d \tau_{q} / d q$ and $f(\alpha)=q \alpha_{q}-\tau_{q}$ (Halsey et al., 1986). It is worth mentioning that in fully developed turbulence, the multifractal spectrum is also related to the scaling exponents of the structure functions through a Legendre transform (Frisch \& Parisi, 1983). This implies that it is often possible to extend theoretical models for the description of the anomalous scaling of the structure functions to the multifractal spectrum, like e.g. the p-model (Meneveau \& Sreenivasan, 1987), which will be used in this paper.

For each sample of solar wind proton density, we estimated the probability measures $\mu(\Delta t)$ and then computed their partition functions $\chi_{q}(\Delta t)$, for 
positive $q \in[0: 5]$, with a step $d q=0.01$. The partition functions for sample $A$ are shown in Figure 5, for the values of the exponents $q>0$, as indicated in the figure legend. Negative orders $q<0$ were also analysed (not shown). However, here we focus on the positive orders only, for which a better determination of the multifractal spectrum was possible. Consistently with the observed double spectral scaling (Šafránková et al., 2015), two separated power-law scaling ranges were identified in the fluid range and in a smaller scale range, respectively above and below the proton gyro-scale. Least-square power-law fits provided the values of the set of exponents $\tau_{q}$ for each of the two ranges, and for each sample. From these, the generalized dimensions $D_{q}$ and the singularity spectrum $f(\alpha)$ were also obtained. One example of these quantities is given in Figure 6 for subsample $A$, for both large and small scale ranges. Deviation from the fractal behavior (i.e. from linear dependence of $\tau_{q}$, and from single-valued $D_{q}$ and $\left.f(\alpha)\right)$ is evident. While the multifractal analysis for all eight samples gives similar results, considerable differences are present between small and large scales. In particular, the small scale quantities indicate in all cases a larger degree of multifractality, as evidenced for example by the broader distribution of singularity exponents.

In order to obtain a quantitative description with one single parameter, two different approaches were used and compared. First, we performed a fit of the scaling exponents $\tau_{q}$ with a p-model, a popular description of intermittency in the framework of a multiplicative, multifractal nonlinear turbulent cascade (Meneveau \& Sreenivasan, 1987). The p-model was originally developed for the description of the energy cascade in Navier-Stokes turbulence. It is a simple representation of the cascade in which the energy at one given position and scale is redistributed unevenly to two smaller scale (or "daughter") structures. The fraction of energy transferred at each step to each daughter structure is given by a cascade of multipliers randomly extracted from a binomial distribution, i.e. $P$ or $1-P$, where $0 \leq P \leq 0.5$ is the parameter that determines the characteristics of the cascade. In this simplistic view, the scaling exponents $\tau_{q}$ are directly related to the value of $P$ through the following relation:

$$
\tau_{q}=-\log _{2}\left[P^{q}+(1-P)^{q}\right] .
$$

The parameter $P$ can be obtained from the fit of the experimental $\tau_{q}$ to equation (4), indicated as lines in Figure 6. Fits of $D_{q}$ and $f(\alpha)$ are obtained using equation (4) in the definitions of those variables. This parameter $P$ is 


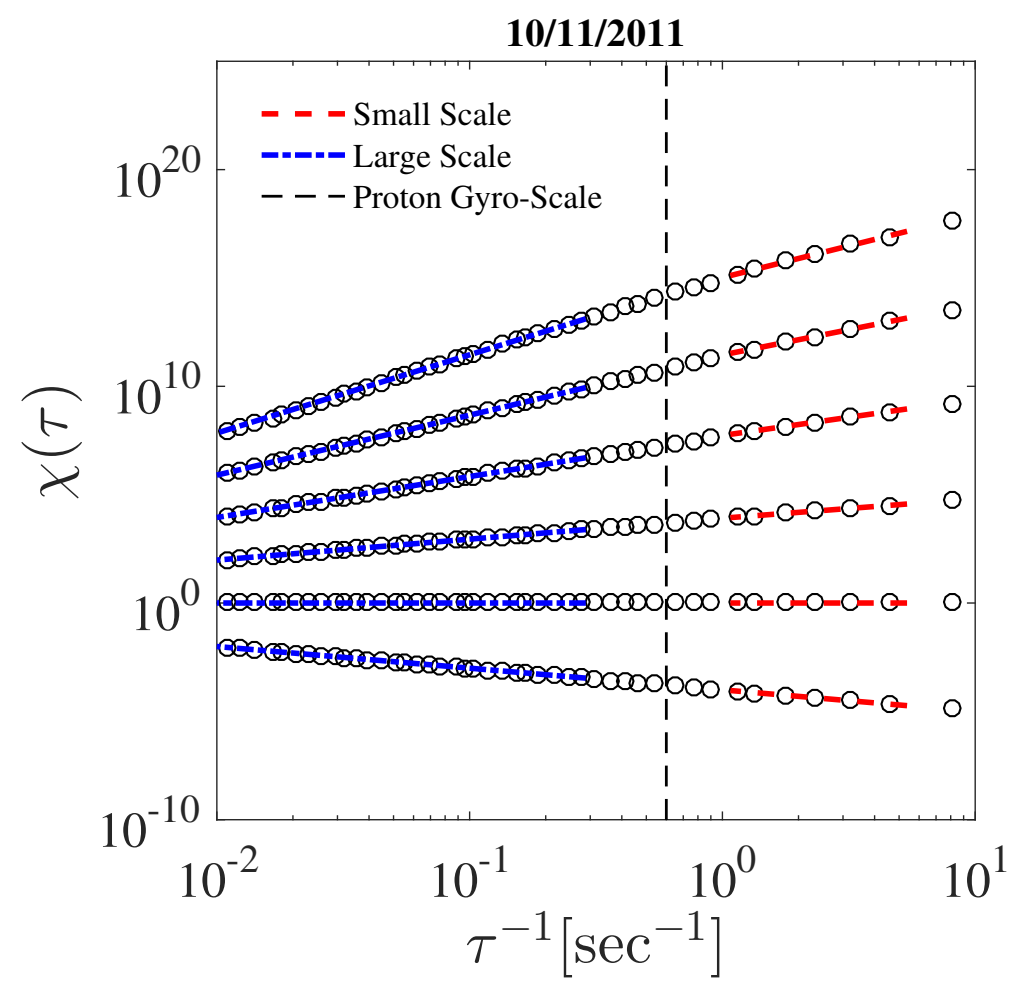

Figure 5: Partition functions $\chi_{q}(\Delta t)$ for sample $A$, for some values of the exponent $q$ as indicated in the legend. Power-law fits in the large scale range (blue dot-dashed lines) and in the small scale ranges (red dashed lines) are also indicated. The vertical line indicates the approximate proton gyro-frequency. 

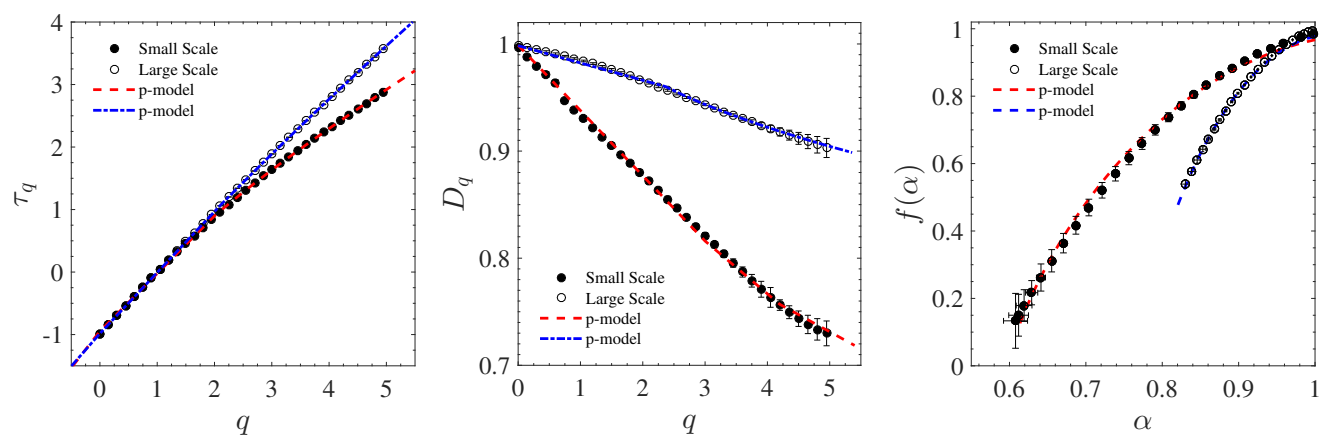

Figure 6: Left panel: the scaling exponents $\tau_{q}$ versus the order $q$, obtained from the fit of the partition functions, eq. (2) at small (black circles) and large (open circles) scales, shown here for sample $A$. Central panel: the corresponding generalized dimensions $D_{q}$ versus $q$. Right panel: the corresponding multifractal spectra $f(\alpha)$ versus the singularity strength $\alpha$ (see text). Lines represent the fits of the quantities $\tau_{q}, D_{q}$ and $f(\alpha)$ with the p-model (4) within the range of large (blue lines) and small (red lines) scales.

thus a good quantitative measure of the deviation from self-similar (or fractal) scaling, i.e. of the degree of multifractality of the system. In particular, $P \simeq 0.5$ is an indication of mono-fractal fields, while smaller values are associated with greater multifractality. The values obtained from the fit, listed in Table 2, confirm the qualitative result that small scales are more multifractal than large scales and depend only weakly on the particular sample.

A different quantitative description of the degree of multifractality was adopted for the description of the radial evolution of magnetic field intermittency in the Heliosphere, making use of Voyager data (Macek et al., 2012). These authors defined a "multifractal index" as the range of values of the singularity exponents $\Delta \alpha=2\left|\alpha_{\max }-\alpha_{\min }\right|$ estimated from the data. Here $\alpha_{\max }$ and $\alpha_{\min }$ indicate the maximum and minimum values of the measured $\alpha$. A larger multifractal index, indicating larger variability of the singularity exponents, corresponds thus to greater multifractality. The multifractal indexes obtained for the eight samples studied here are given in Table 2, along with their average and standard deviation calculated over the different samples. The numbers confirm the general observation that small scale turbulence is more multifractal than in the inertial range. However, the measured values of $\Delta \alpha$ show larger variability than $P$, possibly because $\alpha_{\max }$ and 
$\alpha_{\min }$ are related to the most statistically sensitive part of the data (strongest "burstiness"), and may thus depend on the sample quality.

A direct comparison between the two methods is shown in the top-left panel of Figure 7, where the values of $P$ and $\Delta \alpha$ are scatter-plotted for the eight solar wind samples, indicated by capital (large scale) and small (small scale) letters. Linear regression fits are also superposed in the plots, and all Spearman correlation coefficients are listed in Table 3. The two indicators give consistent results at large scales, and somewhat less consistent values at small scales. In both cases, they are obviously anticorrelated. In the following, we will compare the different parameters with the intermittency measure $P$ and with the alternative measure $\Delta \alpha$.

We have checked the presence of possible correlation between the degree of multifractality $P$ or $\Delta \alpha$ and the spectral index of the density field. The top-right panel of Figure 7 shows for example that there is no evident correlation between $P$ and the corresponding spectral index magnitude $\beta$. This is confirmed by the small correlation coefficients (see Table 3). A different observation holds for the parameter $\Delta \alpha$, where correlations are much larger (not shown, but correlation coefficients are listed in Table 3), suggesting that the finer geometrical nature of the intermittent structures might depend on the spectral properties.

Finally, in order to explore possible differences with alternative estimates of intermittency, we have computed the field kurtosis $K(\Delta t)=\left\langle\Delta n_{p}^{4}\right\rangle /\left\langle\Delta n_{p}^{2}\right\rangle^{2}$, a standard indicator of the deviation from Gaussian statistics (Frisch, 1995). Intermittency is often associated to the value of the kurtosis at the bottom of the corresponding scaling range, which we indicate here as $K_{\max }$. However, a more correct interpretation is to estimate the scaling properties of kurtosis, i.e. the anomalous scaling of the field statistics. This can be done through a power-law fit of the kurtosis $K(\Delta t) \propto \Delta t^{-\gamma}$ in each range where scaling is expected. The kurtosis scaling exponents $\gamma$ can be used as an indicator of the intermittency of the system, and for Navier-Stokes fluids may also be linked to the multifractal properties of turbulence (Frisch \& Parisi, 1983; Castaing et al., 1990; Sorriso-Valvo et al., 2015). A larger exponent indicates faster increase of the kurtosis as the scale decreases, corresponding to a more efficient emergence of localized, intermittent structures in the flow. Figure 8 shows the scale dependence of the kurtosis for one of the samples under study. The general behaviour is an increase toward smaller scales, although a transiton region around the typical proton gyro-scale is present (Chen et al., 2014). The values $K_{\max }$ are highlighted with different markers, and power-law fits 
(in the same ranges where the partition functions $\chi_{q}(\Delta t)$ were fitted to power laws) are superposed as straight lines. Similar results hold for all the other intervals (not shown).

The two bottom panels of Figure 7 show scatter plots of the values of $P$ and $K_{\max }$ (bottom-left) or $\gamma$ (bottom-right) obtained for the eight samples. The first observation is that, while there is a tendency to slightly larger $K_{\max }$ in the small-scale range, the values of $\gamma$ are significantly larger (in absolute values) at large scales, suggesting that the energy cascade is more efficient in generating turbulent structures in the inertial range than in the sub-proton range (Kiyani et al., 2009; Wu et al., 2013; Chen et al., 2014). This may be interpreted as stronger intermittency. However, this indication is in contrast with the behaviour of both $P$ and $\Delta \alpha$, whose values suggest that small scales have larger intermittency. This discrepancy is most probably due to the peculiarities of the solar wind turbulent cascade, and in particular to the presence of a double scaling range. In fact, the small-scale energy cascade starts with non-Gaussian fluctuations and with the presence of highly localized, intermittent structures generated at the bottom of the inertial range cascade. The multifractal measure may capture the degree of morphological complexity of such structures differently from the kurtosis, adding up the effects of the non-homgeneous distribution of the structures due to the inertial range intermittent cascade, and thus providing a more general measure of intermittency. Conversely, the scaling of the small-scale range kurtosis, represented by $\gamma$, is less dependent on the fine details within each structure, and describes better the efficiency of the cascade in accumulating energy in small scale fluctuations. The discrepancy between the two measures can therefore account for the different sensitivity to multiple aspects of the turbulent energy transfer process in solar wind plasma. Similar features were recently observed in the intermittency of numerical simulations of Hall-MHD turbulence (Martin et al., 2014): the intermittent structures were observed to form mostly in the inertial range, while in the Hall-MHD range they changed their fractal dimension by developing a more complex, filamented internal structure. In this sense, the two intermittency parameters give complementary indications that need to be interpreted together for a more complete description.

The scatter plots in Figure 7 show that, in the large scale range, the parameter $P$ and the maximum value of the kurtosis are moderately correlated $\rho\left(P, K_{\max }\right)=0.5$, while a weak correlation (see Table 3 ) is present with the scaling index $\gamma$. The indicators $P$ and $\gamma$ therefore seem to give only 
weakly consistent results in the large scale range. At small scales, correlation between $P$ and $K_{\max }$ is weaker (0.4), similar to the correlation found between $P$ and $\gamma$, with $\rho(P, \gamma)=0.3$ (Table 3). The intermittency indicator $\Delta \alpha$ shows similar correlation as for $P$ at large scale, for both $K_{\max }$ and $\gamma$, and for $K_{\max }$ at small scales. However, a better correlation (0.5) is found at small scales between $\Delta \alpha$ an $\gamma$. This seems to indicate that, while at large scale the p-model parameter could represent a moderately accurate indicator of intermittency, at smaller scale the parameter $\Delta \alpha$ is more useful to assess the deviation from self-similarity.

Note that these results could be affected by the difficult estimation of the field high-order moments (and thus of the kurtosis) through the scaledependent field increments, in particular when the spectral index approaches $\beta \simeq 3$ (Abry et al., 1993; Farge \& Schneider, 2006), or when the scaling ranges are not well extended, and suggests the need for more extended investigation.

\section{Conclusions}

In this work, we have examined the intermittency of small-scale solar wind turbulence by making the first measurement of the multifractal spectrum $f(\alpha)$ of the density fluctuations at scales smaller than the proton gyroscale. We also compared these findings to other measurements of intermittency at small scales, such as the p-model, the kurtosis, and its scaling exponent $\gamma$, as well as similar measurements of the intermittency at larger scales, above the proton gyrocsale.

The results indicate that intermittency is still present in the sub proton gyroscale density fluctuations, which show a higher degree of multifractality than the turbulence at larger scales, i.e., a broader multifractal spectrum (larger $\Delta \alpha$ ). This is in apparent disagreement with previous observations of the density (Chen et al., 2014) and magnetic (Kiyani et al., 2009, 2013; Wu et al., 2013) fluctuations in this range, for which little scale-dependence of the PDFs, or change of the kurtosis is seen. However, our measurements of the scale-dependent kurtosis also reveal greater self-similary in the sub proton gyroscale range, more consistent with these observations.

It seems, therefore, that for the small scale solar wind turbulence, different measures of intermittency can produce different results, possibly due to the different aspects of the fluctuations which they capture. The results may further be complicated by the fact that the small scale fluctuations exists within 

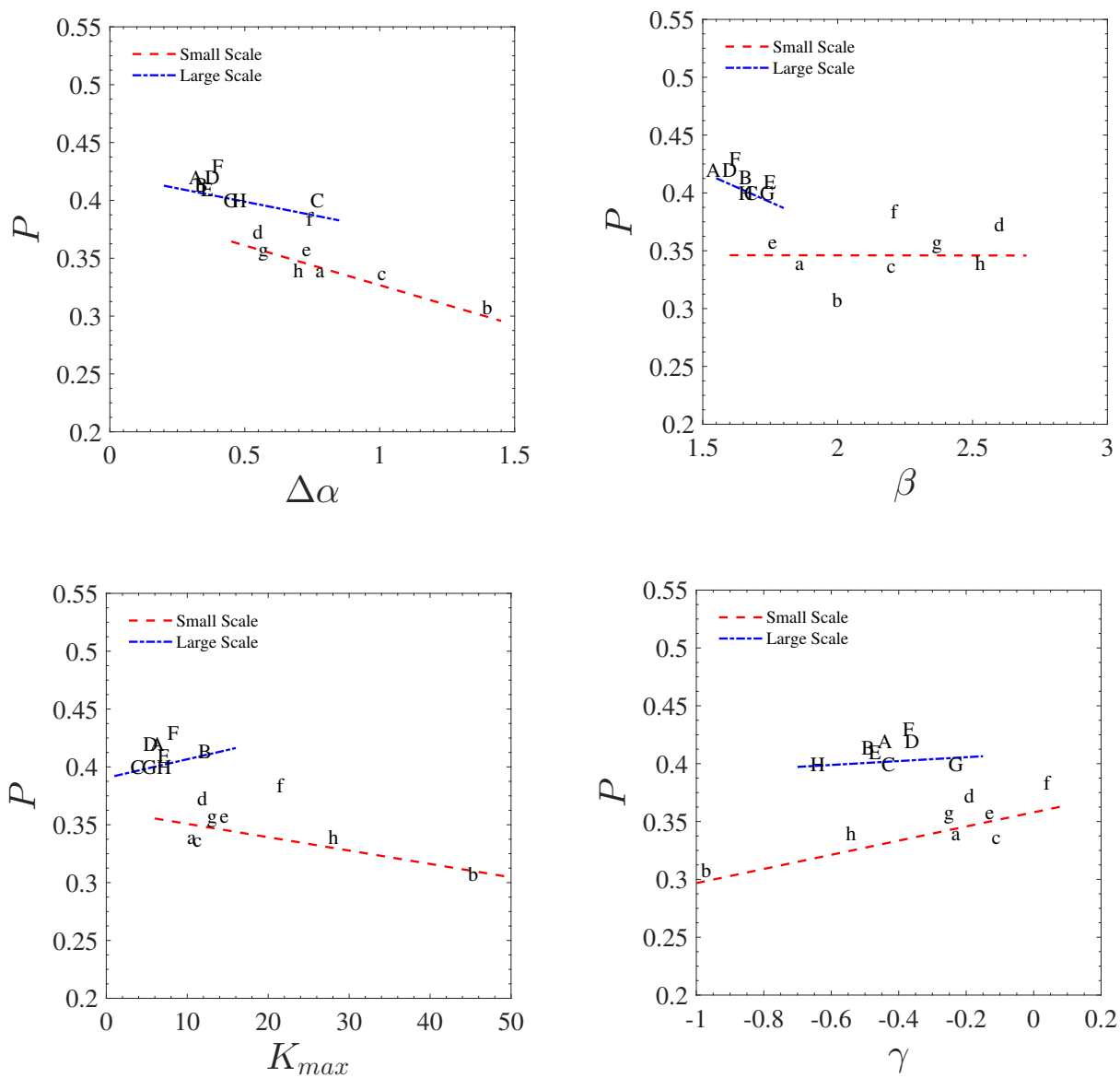

Figure 7: Top-left panel: correlation between $P$ and $\Delta \alpha$ for the eight samples. Top-right panel: correlation between the spectral index $\beta$ and the multifractal parameter $P$ for the small and large scale ranges. Bottom Panels: correlation between the multifractal parameter $P$ and the two kurtosis parameters $K_{\max }$ (left panel) and $\gamma$ (right panel), for the small and large scale ranges. 


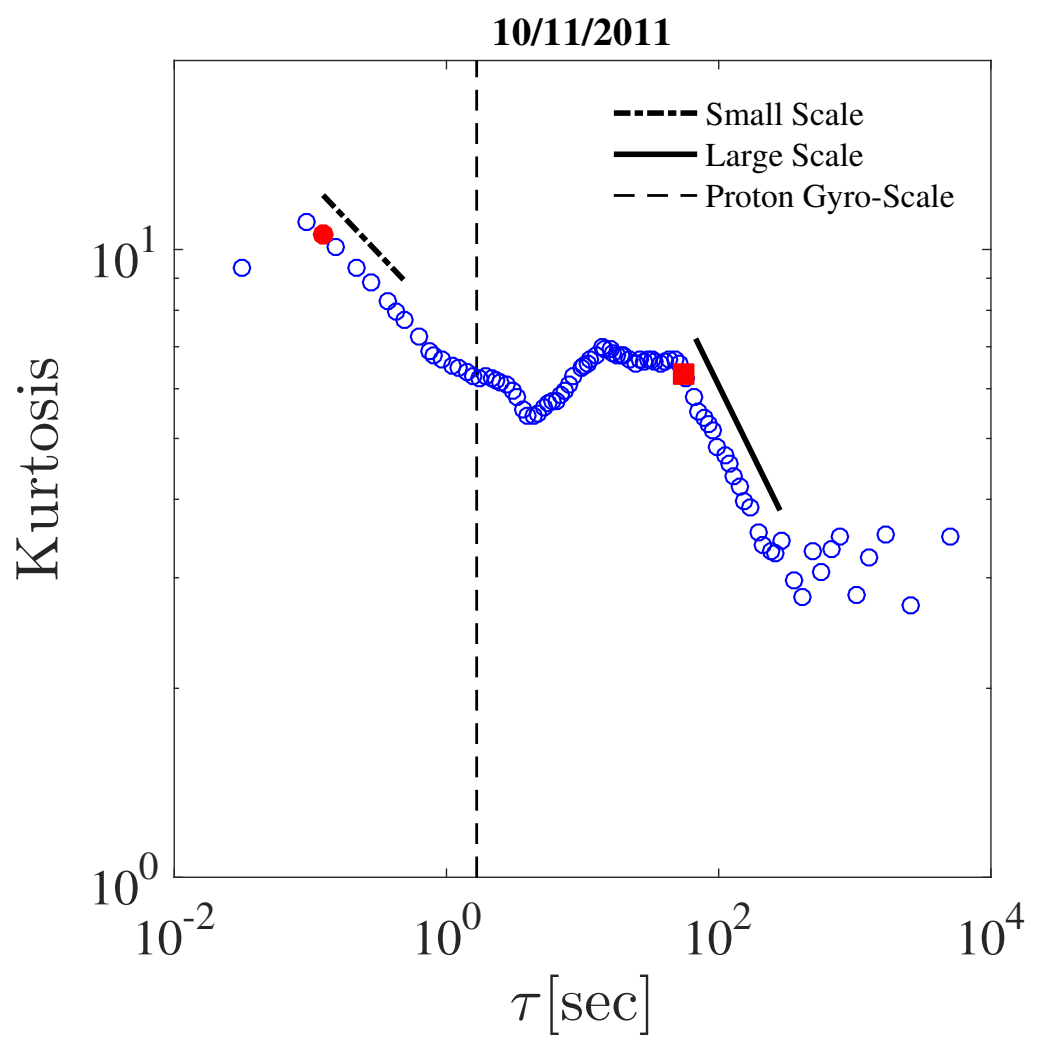

Figure 8: The scale-dependent kurtosis for sample $A$. The values of $K_{\max }$ are highlighted with a red square marker at large scale and with a red bullet at small scales. Power-law fits are superposed as full lines. The vertical line indicates the approximate proton temporal gyroscale. 
the larger MHD scale cascade. The large scale cascade generates structures with a specific nature, morphology (e.g. geometry, fractal dimension, filling factor), and distribution, although in the small-scale range, the nature of the structures generated is different (Martin et al., 2014). Of course, processess of nature other than a fluid nonlinear cascade, occurring at small scales, can also concur to modify the intermittency level. These combined effects may lead to the apparent differences in the various intermittency measures. In general, we have shown that it is important to use multiple estimators to better understand the intermittency at small scales in the solar wind.

Finally, it should be noted that various uncertainties arise when estimating small-scale intermittency, related to the quality of the measurements, the length of the time series, and the spectral properties of the fluctuations (Abry et al., 1993; Farge \& Schneider, 2006; Cho \& Lazarian, 2009; Kiyani et al., 2013). It would, therefore, be of interest, in the future, to obtain higher quality data (e.g. longer records in the pristine solar wind, with better signalto-noise ratio and higher sampling rate) and perform a more comprehensive comparison of different intermittency measures to fully understand the smallscale features of solar wind turbulence.

\section{Acknowledgements}

The research leading to these results has received funding from the European Community's Seventh Framework Programme FP7-PEOPLE-2010IRSES under grant agreement n. 269297/"TURBOPLASMAS" and from ISSI/ISSI-BJ Team 304. LSV acknowledges useful discussion with L. Chevillard and the CNR STM-2014 and STM-2015 programmes. EL acknowledges POR Calabria - FSE 2007/2013. CHKC is supported by an Imperial College Junior Research Fellowship. JS and ZN acknowledge support of the Czech Science Foundation under Contract 16-04956S.

Abry P., Gonçalves P. \& Flandrin P., In IEEE, Int. Conf. on Acoust., Wavelet-based spectral analysis of 1/f processes. Speech and Signal Proc., Minneapolis (US), April 1993 3, Vol. 3, p. 237 (1993).

Alexandrova O., Carbone V., Veltri P. \& Sorriso-Valvo L., Small-scale energy cascade of the solar wind turbulence, Astrophys. J 674, 1153 (2008).

Anselmet F., Gagne Y., Hopfinger E.J. \& Antonia R. A., High-order velocity structure functions in turbulent shear flows, J. Fluid Mech 140, 63 (1984). 
Table 2: The p-model parameter $P$, the multifractal index $\Delta \alpha$, and the spectral index $\beta$ for the eight inervals under study. The average $\langle\cdot\rangle$ and standard deviation $\sigma$ over the eight intervals are aslo given in the last row.

\begin{tabular}{ccccccc}
\hline \hline Interval & \multicolumn{2}{c}{$P$} & \multicolumn{2}{c}{$\Delta \alpha$} & \multicolumn{2}{c}{$\beta$} \\
\hline & small scale & large scale & small scale & large scale & small scale & large scale \\
\hline$A$ & $0.33 \pm 0.01$ & $0.41 \pm 0.01$ & $0.78 \pm 0.1$ & $0.32 \pm 0.09$ & $1.86 \pm 0.01$ & $1.54 \pm 0.01$ \\
$B$ & $0.30 \pm 0.03$ & $0.40 \pm 0.05$ & $1.40 \pm 0.2$ & $0.34 \pm 0.09$ & $2.00 \pm 0.01$ & $1.66 \pm 0.01$ \\
$C$ & $0.33 \pm 0.01$ & $0.39 \pm 0.01$ & $1.01 \pm 0.1$ & $0.77 \pm 0.1$ & $2.20 \pm 0.02$ & $1.68 \pm 0.01$ \\
$D$ & $0.36 \pm 0.03$ & $0.41 \pm 0.01$ & $0.55 \pm 0.1$ & $0.38 \pm 0.1$ & $2.60 \pm 0.01$ & $1.68 \pm 0.03$ \\
$E$ & $0.35 \pm 0.01$ & $0.40 \pm 0.02$ & $0.73 \pm 0.1$ & $0.36 \pm 0.1$ & $1.76 \pm 0.02$ & $1.60 \pm 0.01$ \\
$F$ & $0.37 \pm 0.01$ & $0.42 \pm 0.02$ & $0.74 \pm 0.2$ & $0.40 \pm 0.2$ & $2.21 \pm 0.01$ & $1.75 \pm 0.02$ \\
$G$ & $0.35 \pm 0.01$ & $0.39 \pm 0.02$ & $0.57 \pm 0.2$ & $0.54 \pm 0.1$ & $2.37 \pm 0.01$ & $1.62 \pm 0.01$ \\
$H$ & $0.33 \pm 0.01$ & $0.39 \pm 0.02$ & $0.70 \pm 0.3$ & $0.58 \pm 0.1$ & $2.53 \pm 0.01$ & $1.74 \pm 0.01$ \\
\hline$\langle\cdot\rangle \pm \sigma$ & $0.34 \pm 0.02$ & $0.40 \pm 0.01$ & $0.8 \pm 0.3$ & $0.46 \pm 0.16$ & $2.2 \pm 0.3$ & $1.66 \pm 0.07$ \\
\hline
\end{tabular}


Table 3: The absolute value of the Spearman correlation coefficients and the relative error (the two-sided significance level of its deviance from zero) for the different pairs of parameters.

\begin{tabular}{cccccccc}
\hline \hline & $|\rho(P, \Delta \alpha)|$ & $|\rho(P, \beta)|$ & $\left|\rho\left(P, K_{\max }\right)\right|$ & $|\rho(P, \gamma)|$ & $\left|\rho\left(\Delta \alpha, K_{\max }\right)\right|$ & $|\rho(\Delta \alpha, \beta)|$ & $|\rho(\Delta \alpha, \gamma)|$ \\
\hline large scale & $0.67 \pm 0.10$ & $0.1 \pm 0.8$ & $0.5 \pm 0.2$ & $0.2 \pm 0.7$ & $0.6 \pm 0.1$ & $0.61 \pm 0.1$ & $0.1 \pm 0.9$ \\
small scale & $0.6 \pm 0.2$ & $0.3 \pm 0.5$ & $0.4 \pm 0.4$ & $0.3 \pm 0.5$ & $0.4 \pm 0.4$ & $0.71 \pm 0.07$ & $0.5 \pm 0.2$ \\
\hline
\end{tabular}


Benzi R., Paladin G., Parisi G. \& Vulpiani A., On the multifractal nature of fully developed turbulence and chaotic systems, J. Phys. A: Math. Gen. 17, 3521 (1984).

Bruno R. \& Carbone V., The Solar Wind as a Turbulence Laboratory, Liv. Rev. in Solar Phys. 2 (2013).

Bruno R., Telloni D., Primavera L. et al., Radial evolution of the intermittency of density fluctuations in the fast solar wind, Astrophys. J. 786, 53 (2014).

Burlaga L. F., Multifractal structure of the interplanetary magnetic field near 25 AU, Geophys. Res. Lett. 18, 69 (1991).

Burlaga L. F., Multifractal structure of the interplanetary magnetic field and plasma in recurrent streams at 1 AU, J. Geophys. Res. 97, 4283 (1992).

Burlaga L. F., Multifractal structure of the large-scale heliospheric magnetic field strength fluctuations near 85 AU, Nonlinear Processes Geophys. 11, 441 (2004).

Burlaga L. F., Ness N. F. \& Acuña M. H., Multiscale structure of magnetic fields in the heliosheath, J. Geophys. Res. 111, A09112 (2006).

Burlaga L. F. \& Ness N. F., Sectors and large-scale magnetic field strength fluctuations in the heliosheath near 110 AU: Voyager 1, 2009, Astrophys. J. 725, 1306 (2010).

Castaing B., Gagne Y., \& Hopfinger E., Velocity probability density functions of high Reynolds number turbulence, Physica D 46, 177 (1990).

Chhabra A. B., Meneveau C., Jensen R. V. \& Sreenivasan K. R. Direct determination of the $f(\alpha)$ singularity spectrum and its application to fully developed turbulence, Phys. Rev. A 40, 5284 (1989).

Chen C. H. K., Bale S. D., Salem C., Mozer F. S., Frame Dependence of the Electric Field Spectrum of Solar Wind Turbulence, Astrophys. J. Lett. 737, L41 (2011).

Chen C. H. K., Salem C. S., Bonnell J. W., Mozer F. S., Bale S. D., Density Fluctuation Spectrum of Solar Wind Turbulence between Ion and Electron Scales, Phys. Rev. Lett. 109, 035001 (2012). 
Chen C. H. K., Sorriso-Valvo L., Šafránková J. \& Němeček Z., Intermittency of Solar Wind Density Fluctuations From Ion to Electron Scales, Astrophys. J. Lett. 789, L8 (2014).

Cho J. \& Lazarian A., Simulations of electron magnetohydrodynamic turbulence, Astrophys. J. 701, 236 (2009).

Coleman P., Turbulence, Viscosity, and Dissipation in the Solar-Wind Plasma, Astrophys. J. 153, 371 (1968).

Farge M. \& Schneider K., Wavelets: applications to turbulence, in Encyclopedia of Mathematical Physics, ed. J. P. Françoise, G. Naber \& T. S. Tsun (Elsevier), 408 (2006).

Frisch U., Turbulence. The legacy of A. N. Kolmogorov. Cambridge University Press, Cambridge, UK (1995).

Frisch U. \& Parisi G., On the singularity structure of fully developed turbulence, in Turbulence and Predictability of Geophysical Flows and Climate Dynamics, Varenna Summer School LXXXVII, 84 (1983).

Grassberger P. \& Procaccia I., Characterization of Strange Attractors, Phys. Rev. Lett. 50, 346 (1983).

Halsey T. C., Jensen M. H., Kadanoff L. P., Procaccia I. \& Shraiman B. I., Fractal measures and their singularities: The characterization of strange sets, Phys. Rev. A 33, 1141 (1986).

Hnat, B., Chapman, S. C., \& Rowlands, G., Intermittency, scaling, and the Fokker-Planck approach to fluctuations of the solar wind bulk plasma parameters as seen by the WIND spacecraft, Phys. Rev. E 67, 056404 (2003).

Hnat, B., Chapman, S. C., \& Rowlands, G., Compressibility in Solar Wind Plasma Turbulence, Phys. Rev. Lett. 94, 204502 (2005).

Kiyani K., Chapman S. C. \& Hnat B., Extracting the scaling exponents of a self-affine, non-Gaussian process from a finite-length time series, Phys. Rev. E, 74, 051122 (2006). 
Kiyani K. H., Chapman S. C., Khotyaintsev Yu. V., Dunlop M. W. \& Sahraoui F., Global Scale-Invariant Dissipation in Collisionless Plasma Turbulence, Phys. Rev. Lett. 103, 075006 (2009).

Kiyani K. H., Chapman S. C., Sahraoui F. et al., Enhanced Magnetic Compressibility and Isotropic Scale Invariance at Sub-ion Larmor Scales in Solar Wind Turbulence, Astrophys. J. 7763, 10 (2013).

Kolmogorov A. N., The local structure of turbulence in an incompressible viscous fluid forvery large Reynolds numbers, C.R. Acad. Sci., USSR 30, 301 (1941).

Leonardis E., Sorriso-Valvo L., Valentini F., Servidio S., Carbone F., \& Veltri P., Multifractal scaling and intermittency in hybrid Vlasov-Maxwell simulations of plasma turbulencePhys. of Plasmas 23, 022307 (2016).

Macek W. M., Modeling Multifractality of the Solar Wind, Space Sci. Rev. 122, 329 (2006).

Macek W. M., Multifractality and intermittency in the solar wind, Nonlin. Processes Geophys. 14, 695 (2007).

Macek W. M. \& Szczepaniak, Generalized two-scale weighted Cantor set model for solar wind turbulence, Geophys. Res. Lett. 35 L02108, (2008)

Macek W. M. \& Wawrzaszek A., Evolution of asymmetric multifractal scaling of solar wind turbulence in the outer heliosphere, J. Geophys. Res. 114, 3108 (2009).

Macek W. M, Wawrzaszek A. \& Carbone V., Observation of the multifractal spectrum at the termination shock by Voyager 1, Geophys. Res. Lett. 38, L19103 (2011).

Macek W. M, Wawrzaszek A. \& Carbone V., Observation of the multifractal spectrum in the heliosphere and the heliosheath by Voyager 1 and 2, J. Geophys. Res. 117, A12101 (2012).

Macek W. M, Wawrzaszek A. \& Burlaga L. F., Multifractal Structures Detected by Voyager 1 at the Heliospheric Boundaries, Astrophys. J. Lett. 793, L30 (2014). 
Malara F., Primavera L. \& Veltri P., Nonlinear evolution of the parametric instability: numerical predictions versus observations in the heliosphere, Nonlin. Processes Geophys. 8, 159 (2001).

Mandelbrot B., The Fractal Geometry of Nature, Freeman, San Francisco (1982).

Marsch E. \& Tu C.-Y., Spectral and spatial evolution of compressive turbulence in the inner solar wind, J. Geophys. Res. 95, 11945 (1990).

Marsch E., Tu C.-Y. \& Rosenbauer H., Multifractal scaling of the kinetic energy flux in solar wind turbulence, Ann. Geophys. 14, 259 (1996).

Marsch E. \& Tu C.-Y., Intermittency, non-Gaussian statistics and fractal scaling of MHD fluctuations in the solar wind, Nonlin. Processes in Geophys. 4, 101 (1997).

Martin L. N., De Vita G., Sorriso-Valvo L., Dmitruk P., Nigro G., Primavera L., Carbone V., Cancellation properties in Hall magnetohydrodynamics with a strong guide magnetic field, Phys. Rev. E 88, 063107 (2013).

Meneveau C. \& Sreenivasan K. R., Simple multifractal cascade model for fully developed turbulence, Phys. Rev. Lett. 59, 1424 (1987).

Montgomery D., Brown M. R. \& Matthaeus W. H., Density fluctuation spectra in magnetohydrodynamic turbulence, J. Geophys. Res. 92, 282 (1987).

Ott, E., Chaos in Dynamical Systems, Cambridge Univ. Press, Cambridge, U. K. (1993).

Paladin G. \& Vulpiani A., Anomalous Scaling Laws in Multifractals objects, Phys. Reports 156, 147 (1987).

Primavera L., Malara F. \& Veltri P., Parametric instability in the solar wind: numerical study of the nonlinear evolution, in AIP Conf. Proc. 679, SolarWind Ten, ed. M. Velli, R. Bruno \& F. Malara (Melville, NY: AIP), 505 (2003).

Riazantseva M. O. \& Zastenker G. N., Intermittency of Solar Wind Density Fluctuations and Its Relation to Sharp Density Changes, Cosmic Research, 46, 1, Pleiades Publishing, Ltd. (2008). Original Russian Text published in Kosmicheskie Issledovaniya, 2008, Vol. 46, No. 1, pp. 39. 
Šafránková J., Němeček Z., Prěch L. \& Zastenker G. N., on Kinetic Scale in the Solar Wind Observed, Phys. Rev. Lett. 110, 025004 (2013a).

Šafránková J., Němeček Z. et al., Fast Solar Wind Monitor (BMSW): Description and First Results, Space Sci. Rev. 175, 165 (2013b).

Šafránková J., Němeček Z. et al., Solar Wind Density Spectra around the Ion Spectral Break, Astrophys. J. 803, 107 (2015).

Shaikh D. \& Zank G. P., The turbulent density spectrum in the solar wind plasma, Mon. Not. R. Astron. Soc. 402, 362 (2010).

She Z.-S. \& Lévêque E., Universal scaling laws in fully developed turbulence, Phys. Rev. Lett. 72, 336 (1994).

Sorriso-Valvo L., Carbone V., Veltri P., Consolioni G. \& Bruno R., Intermittency in the solar wind turbulence through probability distribution functions of fluctuations, Geophys. Res. Lett. 26, 1801 (1999).

Sorriso-Valvo L., Carbone V., Giuliani P. et al., Intermittency in plasma turbulence, Planetary and Space Sci. 49, 1193 (2001).

Sorriso-Valvo L., Marino R., Lijoi L. et al., Self-consistent Castaing distribution of solar wind turbulent fluctuations, Astrophys. J. 807, 86 (2015).

Sreenivasan K. R., Fractals and multifractals in fluid turbulence, Ann. Rev. Fluid. Mech. 23539 (1991).

Tu C.-Y. \& Marsch E., MHD structures, waves and turbulence in the solar wind: Observations and theories, Space Science Reviews 73, 1 (1995).

Yordanova E., Grzesiak M., Wernik A. W., Popielawska B. \& Stasiewicz K., Multifractal structure of turbulence in the magnetospheric cusp, Ann. Geophys. 22, 2431 (2004).

Wawrzaszek A., Echim M., Macek W. M, \& Bruno R., Evolution of Intermittency in the Slow and Fast Solar Wind beyond the Ecliptic Plane, Astrophys. J. Lett. 814, L19 (2015).

Wu P., Perri S., Osman K. et al., Intermittent heating in solar wind and kinetic simulations, Astrophys. J. Lett. 763, L30 (2013). 